\title{
On the ballistic performance of metallic materials
}

\author{
B SRIVATHSA and N RAMAKRISHNAN* \\ Computer Simulation Centre, Defence Metallurgical Research Laboratory, Kanchanbagh, \\ Hyderbad 500058 , India \\ (Communicated by Professor P Rama Rao) \\ MS received 6 November 1996
}

\begin{abstract}
The paper presents a ballistic performance index for metallic armour materials in terms of the commonly determined mechanical properties such as strength and modulus. The index is derived using an energy-balance approach, where the kinetic energy of the projectile is assumed to be absorbed by the elastic and the plastic deformation involved in the penetration process as well as the kinetic energy imparted to the target material during deformation. The derivation assumes two distinct stages to exist during the penetration of the projectile. At the striking face of the armour, the material is assumed to flow radially in a constrained deformation region but longitudinally at the rear surface leading to typically observed bulging of the armour without constraint. The index is validated using the available experimental and empirical data obtained in the case of small arm projectiles for an impact velocity of about 800 $\mathrm{m} / \mathrm{sec}$. This index is expected to facilitate the development of metallic armour, since the number of the ballistic experiments can be reduced significantly and only the promising materials need to be considered.
\end{abstract}

Keywords. Terminal ballistics; mechanical properties; metallic armour.

\section{Introduction}

The ballistic resistance of an armour material is normally characterized in terms of the reciprocal of the areal density of the target material that is required to arrest a particular type of projectile striking with a specific velocity. In such development activities, invariably, a large number of experiments are carried out for different alloy compositions and heat treatment processes. This is inevitable, since comprehensive knowledge does not exist about the correlation between the mechanical properties and the ballistic performance. Even an approximate correlation is expected to greatly facilitate the development of armour, since substantial knowledge on the mechanical properties is routinely available for various alloys. The aim of this paper is to arrive at such a ballistic index. For this purpose we have to take into account the various mechanisms that operate during the penetration of the projectile as well as the effect of various mechanical properties on the energy-absorption process. It is in this context, that the earlier investigations are first briefly reviewed.

Various models have been used in the past in determining the energy absorbed by an armour in arresting a projectile. Taylor (1948a) established the work done by a projectile in penetrating a target, assuming a radial expansion of a cylindrical hole of diameter equal to that of the projectile under plane strain condition. Thompson (1955) improved the model of Taylor (1948a) by including the work done for dishing the material when struck by non-deforming projectiles. Averbuch and Bodner (1974) investigated the perforation of a plate by a non-deforming projectile, assuming a three-stage model. In

*Author for correspondence 
the first stage, the motion of the projectile is resisted by the radial plastic flow and the inertial resistance of the target material, while in the second stage, a plug is formed by shear failure resulting in the ejection of the plug in the last stage. A refined method of the above was presented by Ravid and Bodner (1983) in which a two-dimensional model is assumed with five stages of penetration, namely (i) dynamic plastic penetration, (ii) bulge formation, (iii) bulge advancement, (iv) plug formation and (v) the exit of the projectile. In an analysis of high speed impact, Tate (1977) used the constrained-plasticflow approach. Backman and Goldsmith (1978) studied the dominant modes of failure that may occur during the process of penetration and perforation of various targets. Landkof and Goldsmith (1985) determined the energy absorbed in the perforation of thin metallic targets by cylindro-conical projectiles based on a plastic hinge theory for studying the bending of the petals. A unified theory of penetration was proposed by James (1987) in which the theories of shaped charge jets and KE projectiles were integrated. In the perforation of aluminium plates with conical-nosed rods Forrestal et al (1987) considered the radial effects for computing the volume participating in the elastic and plastic deformation.

Although the mechanics of penetration has been studied extensively, only few have focused their attention on the effect of mechanical properties on the ballistic performance. Tabor (1948) considered the effective pressure to be three times the ultimate strength in his calculations. Effect of Young's modulus on shock loading in $2024 \mathrm{Al}$ was discussed by Sinha et al (1977). Tate (1977) assumed the flow strength to be three times that of the measured yield strength. Woodward (1978) described the penetration of a conical projectile using the work done expressed in terms of the flow stress. Although, evaluation of the performance of the projectile on plate targets have been discussed in terms of the fiow stress by many investigators (Bishop et al 1945; Taylor 1948b; Woodward 1978; Corran et al 1983; Landkof and Goldsmith 1985; Forrestal 1986; Randin and Goldsmith 1988), quite a few have observed that merely increasing the mechanical strength does not lead to improved ballistic resistance (Bishop et al 1945; Thornpson 1955; Recht 1964; Abbot 1972; Wingrove and Wulf 1973; Tate 1977, 1980; Jane and Zukas 1978; Longcope and Forrestal 1983). This observation essentially contains significance of the other mechanical properties in influencing the ballistic resistance of the material.

Since our focus is to understand the effect of commonly employed mechanical properties such as yield strength, ultimate strength, percentage elongation and elastic modulus on the ballistic performance, we have adopted a different approach, although our energy-absorption model is broadly similar to the earlier ones. While earlier investigators focused their attention on the non-material parameters such as projectile and target geometry and the ballistic parameters such as velocity, our emphasis is on the material related ones. In addition, it should be noted that this exercise is not aimed at establishing the ballistic limit but establishing the ballistic quality of typical metallic armour materials.

\section{The model}

In this model, a target of finite thickness is divided into two regions as shown in figure 1a. At the striking face of the target, the region (I) is assumed to be in plane strain condition and the material flows only in the radial direction. At the other end (II), the material is assumed to bulge in the process of reducing the projectile velocity to zero. The relative widths of these two regions are computed by a typically observed velocity 

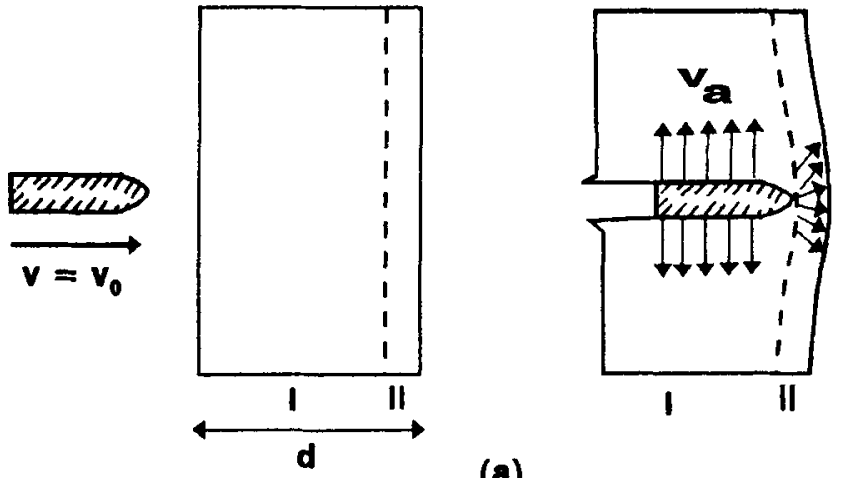

(a)

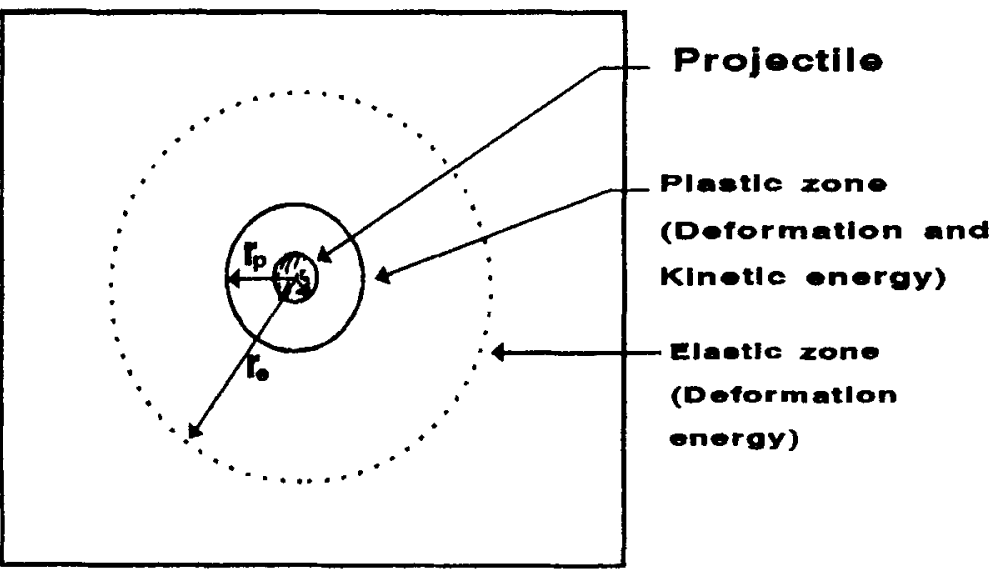

(b)

Figure 1. Schematic diagram showing the penetration mechanism assumed in the model. (a) Constrained (I) and unconstrained (II) regions and (b) elastic and plastic zones.

decay of the projectile and this aspect is explained in $\S 2.1$. The kinetic energy of the striking projectile is assumed to be absorbed mainly in three different modes, as shown in figure $1 \mathrm{~b}$ : (a) the elastic deformation of the material surrounding the projectile, (b) the plastic deformation of the material surrounding the projectile and (c) the kinetic energy imparted to the target material around the target-projectile interface.

The total energy absorbed in each of the above cases is determined as the product of the energy absorbed per unit volume and the volume participating in the respective mode of deformation. The cylindrical volume that participates in each mode is computed using the distance covered by the elastic or the plastic wave, as the case may be, in the radial direction from the line of penetration as shown in figure $1 \mathrm{~b}$. For computing the volume that participates in a specific deformation process, one has to consider the radial spread of the wave front, propagating from the target-projectile interface. When a conical-nosed projectile with a semi-nose angle of about $45^{\circ}$ penetrates any homogeneous material with a velocity of $v$, it imparts a particle velocity of the same magnitude to the target material but in the radial direction. Since there is a gradual reduction of velocity with penetration, ideally, the particle velocity imparted also varies in a similar fashion. A rigorous approach 
is indeed possible where the particle velocity is chosen depending on the location along the thickness of the target. However, this method needs numerical support and the ballistic index cannot be expressed in a simple and explicit form. Therefore, for the sake of simplicity we have assumed a constant representative particle velocity $\left(v_{a}\right)$ for the entire thickness of the target, equal to one half of the striking velocity, similar to the model presented by Taylor (1948a).

\subsection{Calculation of the relative widths of regions $I$ and $I I$}

Let $\alpha_{1}$ and $\alpha_{2}$ be the fractional widths of regions I and II respectively. It is common practice to assume the retardation $(v \mathrm{~d} v / \mathrm{d} x)$ of the penetrating projectile to be a function of the velocity itself. While some investigators have expressed it as a linear function of momentum $(m v)$, some others have used the kinetic energy $\left(1 / 2 m v^{2}\right)$ instead. However, in the real case, it can be assumed to be a combination of these two and it can be expressed as,

$$
v \frac{\mathrm{d} v}{\mathrm{~d} x}=-A v^{3 / 2}
$$

where $v$ is the velocity and $A$ is a constant parameter.

On integrating (1) for $v=v_{0}$ to $v_{x}$, we get

$$
2\left[\sqrt{v_{0}}-\sqrt{v_{x}}\right]=A x,
$$

where $v_{0}$ is the striking velocity, $v_{x}$ the velocity of the projectile after penetrating a depth of $x$ in the target in the direction of penetration. Determining the value of constant $A$ by imposing $v_{x}=0$ for $x=d$ (where $d$ is the total thickness of the target), and then by rearranging $(2)$, we get

$$
1-\frac{\sqrt{v_{x}}}{\sqrt{v_{0}}}=\frac{x}{d}=\alpha_{x},
$$

where $\alpha_{x}$ represents the fractional thickness needed to reduce the projectile velocity to $v_{x}$. The first region (I) is assumed to extend up to a depth where the penetrating projectile just ceases to make a dent. The minimum stress required to produce the dent is about 2.6 times $\sigma_{y}$ (Eichelberger 1956; Allen and Rogers 1961), where $\sigma_{\mathrm{y}}$ is the yield stress of the material. Stress generated by a particle velocity $v$ is (Kinslow 1970)

$$
\sigma=\rho v\left(v+c_{\mathrm{e}}\right)
$$

where $\rho$ the density, $c_{c}$ the longitudinal speed of the elastic wave. Assuming the stress at the interface between I and II to be $2 \cdot 6 \sigma_{y}$, we get

$$
\rho v_{1}\left(v_{1}+c_{\mathrm{e}}\right)=2 \cdot 6 \sigma_{y}
$$

where $v_{1}$ is the velocity of the projectile at the interface. On simplifying (5) for $v_{1}$ we get

$$
v_{1}=\frac{-\rho c_{\mathrm{e}}+\sqrt{\left[\rho^{2} c_{\mathrm{e}}^{2}+10 \cdot 4 \rho \sigma_{\mathrm{y}}\right]}}{2 \rho} .
$$


Substituting $v_{1}$ for $v_{x}$ in (3) we get $\alpha_{1}$ as,

and

$$
\alpha_{1}=1-\frac{\sqrt{v_{1}}}{\sqrt{v_{0}}}
$$

$$
\alpha_{2}=1-\alpha_{1} \text {. }
$$

\subsection{Elastic energy calculations}

The elastic energy absorbed in the constrained(I) and the unconstrained(II) regions are different and these have to be computed separately.

2.2a Constrained zone: The material undergoes a bulk strain in region I. The energy per unit volume absorbed in this case is given by

$$
=\frac{1}{2} K\left[\frac{\Delta V}{V}\right]^{2}
$$

where $K$ is the bulk modulus and $\Delta V / V$ represents the volume strain.

$$
K=c_{\mathrm{b}}^{2} \rho,
$$

where $c_{b}$ is the bulk speed of the elastic wave. The volume strain can be shown to be

$$
\frac{\Delta V}{V}=\frac{r_{0}^{2}}{r_{e}^{2}},
$$

where $r_{0}$ is the radius of the projectile and $r_{\mathrm{e}}$ the radial distance covered by the elastic wave front (figure $1 \mathrm{~b}$ ).

$$
r_{\mathrm{e}}=\left(c_{\mathrm{b}}+v_{\mathrm{a}}\right) t
$$

where $v_{\mathrm{a}}$ is the representative particle speed as described in $\S 2$ and $t$ denotes time.

$$
r_{0}=v_{\mathrm{a}} t
$$

Combining (9), (10), (11) and (12) and substituting in (8) we get the energy per unit volume as

$$
=\frac{1}{2} c_{\mathrm{b}}^{2} \rho\left[\frac{v_{\mathrm{a}}}{c_{\mathrm{b}}+v_{\mathrm{a}}}\right]^{4} .
$$

The cylindrical volume that participates in this deformation is

$$
=\Pi\left(c_{\mathrm{b}}+v_{\mathrm{a}}\right)^{2} t^{2} \alpha_{1} d
$$

Hence the total elastic energy absorbed in the constrained region(I) can be expressed as

$$
\psi_{\mathrm{EL}}^{\mathrm{I}}=\pi t^{2} \rho \mathrm{d} v_{\mathrm{a}}^{4}\left[\frac{\alpha_{1}}{2\left(1+k_{\mathrm{b}}\right)^{2}}\right],
$$

where $k_{\mathrm{b}}$ is a dimensionless parameter defined as

$$
k_{\mathrm{b}}=\frac{v_{\mathrm{a}}}{c_{\mathrm{b}}}
$$


$2.2 \mathrm{~b}$ Unconstrained zone: The energy absorbed in region II is determined using a similar procedure followed in $\S 2.2 \mathrm{a}$ except that the appropriate energy per unit volume and the volume participation are considered.

$$
\text { Energy per unit volume }=\frac{1}{2}\left[\frac{\sigma_{y}^{2}}{E}\right],
$$

where $\sigma_{\mathrm{y}}$ is the yield strength and $E$ the elastic modulus.

Volume participating in this deformation is

$$
=\pi\left(c_{\mathrm{c}}+v_{\mathrm{a}}\right)^{2} t^{2} \alpha_{2} d .
$$

Hence the total elastic energy absorbed in the unconstrained region (II) is,

$$
\psi_{\mathrm{EL}}^{\mathrm{II}}=\pi t^{2} \rho \mathrm{d} v_{\mathrm{a}}^{2}\left[\frac{\alpha_{2} k_{\gamma}^{2}\left(1+k_{\mathrm{e}}\right)^{2}}{2 k_{\mathrm{j}}^{2}}\right] .
$$

The elastic longitudinal wave speed

where

$$
c_{\mathrm{e}}=k_{\gamma} /\left[\frac{E}{\rho}\right]
$$

$$
k_{y}=\sqrt{\left[\frac{1-\gamma}{(1-2 \gamma)(1+\gamma)}\right]}
$$

where $\gamma$ is the Poisson's ratio of the material, and $k_{\mathrm{j}}$ (Johnson 1972) and $k_{\mathrm{e}}$ are dimensionless parameters given as

$$
k_{\mathrm{j}}=\frac{\rho v_{\mathrm{a}}^{2}}{\sigma_{\mathrm{y}}} ; \quad k_{\mathrm{e}}=\frac{v_{\mathrm{a}}}{c_{\mathrm{e}}} .
$$

\subsection{Plastic energy calculations}

The energy absorbed by the plastic deformation is also computed in a similar way as described in $\$ 2.2 \mathrm{a}$ except that the elastic parameters are replaced by the plastic ones. In addition, it is to be noted that there is no need for calculating the energy absorbed by plastic flow for two regions separately, since we need to take only the plastic shear strain into account.

Plastic energy absorbed per unit volume, which is the area under a typical bilinear stress-strain curve, is expressed as

$$
=\sigma_{\mathrm{y}} \varepsilon+\frac{1}{2} E_{\mathrm{p}} \varepsilon^{2},
$$

where $\sigma_{y}$ is the yield stress, $\varepsilon$ the plastic strain and $E_{\mathrm{p}}$ the plastic modulus. Volume participating in the deformation is

$$
=\pi\left(c_{\mathrm{p}}+v_{\mathrm{a}}\right)^{2} t^{2} d .
$$

Hence the total energy due to the plastic deformation is

$$
\psi_{\mathrm{PL}}^{\mathrm{I}, \mathrm{II}}=\pi t^{2} \rho \mathrm{d} v_{\mathrm{a}}^{4}\left[\frac{1}{k_{\mathrm{j}}}\left(1+\frac{1}{k_{\mathrm{p}}}\right)+\frac{1}{2 k_{\mathrm{p}}^{2}}\right],
$$


where

$$
k_{\mathrm{j}}=\frac{\rho v_{\mathrm{a}}^{2}}{\sigma_{\mathrm{y}}}, \quad k_{\mathrm{p}}=\frac{v_{\mathrm{a}}}{c_{\mathrm{p}}}, \quad E_{\mathrm{p}}=c_{\mathrm{p}}^{2} \rho \quad \text { and } \quad \varepsilon=\frac{v_{\mathrm{a}}}{c_{\mathrm{p}}+v_{\mathrm{a}}}
$$

\subsection{Kinetic energy imparted to the material}

The kinetic energy imparted to the target material is significant only in the plastic zone and turns out to be negligible in the purely elastic zone. Since the particle velocity imparted to the target material is $v_{a}$, as discussed in $\S 2.2$, the kinetic energy imparted per unit volume to the target material is,

$$
=\frac{1}{2} \rho v_{\mathrm{a}}^{2} \text {. }
$$

The volume that participates in this process is same as that given in (22) and therefore the total kinetic energy imparted to the target material is

where

$$
\psi_{\mathrm{ke}}^{\mathrm{I}, \mathrm{II}}=\pi t^{2} \rho \mathrm{d} v_{\mathrm{a}}^{4}\left[\frac{1}{2}\left(1+\frac{1}{k_{\mathrm{p}}}\right)^{2}\right],
$$

$$
k_{\mathrm{p}}=\frac{v_{\mathrm{a}}}{c_{\mathrm{p}}}
$$

\section{The ballistic performance index}

The total energy absorbed by all the three modes [from (15),(19),(23) and (24)] is

$$
\psi=\psi_{\mathrm{EL}}^{\mathrm{I}}+\psi_{\mathrm{EL}}^{\mathrm{II}}+\psi_{\mathrm{PL}}^{\mathrm{IIII}}+\psi_{\mathrm{KE}}^{\mathrm{I}, \mathrm{II}}
$$

The total energy absorbed per unit areal density $(\rho d)$ is

$$
\begin{aligned}
\frac{\psi}{\rho d}=\pi t^{2} v_{\mathrm{a}}^{4} & {\left[\frac{\alpha_{1}}{2\left(1+k_{\mathrm{b}}\right)^{2}}+\alpha_{2} \frac{\left(1+k_{\mathrm{e}}\right)^{2} k_{\gamma}^{2}}{2 k_{\mathrm{j}}^{2}}+\frac{1}{k_{\mathrm{j}}}\left(1+\frac{1}{k_{\mathrm{p}}}\right)+\frac{1}{2 k_{\mathrm{p}}^{2}}\right.} \\
& \left.+\frac{1}{2}\left(1+\frac{1}{k_{\mathrm{p}}}\right)^{2}\right] .
\end{aligned}
$$

In (26), it can be seen that the terms inside the square bracket exclusively correspond to the mechanical properties and the velocity of interest, making it ideally suitable for characterizing the ballistic quality. Thus the ballistic merit can be expressed as,

$$
\begin{aligned}
\phi= & {\left[\frac{\alpha_{1}}{2\left(1+k_{\mathrm{b}}\right)^{2}}+\alpha_{2} \frac{\left(1+k_{\mathrm{e}}\right)^{2} k_{y}^{2}}{2 k_{\mathrm{j}}^{2}}+\frac{1}{k_{\mathrm{j}}}\left(1+\frac{1}{k_{\mathrm{p}}}\right)+\frac{1}{2 k_{\mathrm{p}}^{2}}\right.} \\
& \left.+\frac{1}{2}\left(1+\frac{1}{k_{\mathrm{p}}}\right)^{2}\right]
\end{aligned}
$$

On the right hand side of (27) the first two terms represent the elastic components, the third and the fourth represent the plastic components and the last term corresponds to the kinetic energy component. Since all the terms appearing on the right hand side of (27) are dimensionless parameters, $\phi$ is also a dimensionless parameter. In all our 
Table 1. Material properties*.

\begin{tabular}{lcccc}
\hline Material & $\begin{array}{c}\text { Density }(\rho) \\
\left(\mathrm{kg} \cdot \mathrm{m}^{-3}\right)\end{array}$ & $\begin{array}{c}\text { Yield strength } \\
\left(\sigma_{\mathrm{y}}(\mathrm{Mpa})\right)\end{array}$ & $\begin{array}{c}\text { Tensile strength } \\
\left(\sigma_{\mathrm{u}}(\mathrm{Mpa})\right)\end{array}$ & $\%$ Elongation \\
\hline Lead & 11340 & 15 & 38 & 50 \\
Copper & 8940 & 275 & 372 & 20 \\
Mild steel & 7800 & 325 & 691 & 35 \\
Aluminium & 2720 & 130 & 217 & 20 \\
Steel-A & 7800 & 1068 & 1210 & 11 \\
Ti-alloy & 4550 & 990 & 1050 & 15 \\
Magnesium & 1760 & 200 & 310 & 15 \\
Al-2024 & 2770 & 345 & 565 & 17 \\
Steel-B & 7800 & 1610 & 1860 & 11 \\
Al-7049 & 2790 & 455 & 585 & 11 \\
\hline
\end{tabular}

* These values are taken from Metals Handbook (1993), Smithells (1983) and Madhu (1994)

results, we have normalized this index with respect to that of mild steel for a quick appraisal of the ballistic quality.

\section{Validation and discussion}

For testing the usefulness of the proposed index, we considered ballistic performance of ten materials listed in table 1. The experimental data pertaining to Mild steel, Aluminum, Steel-A, Steel-B and Al-7049 were obtained from the published results of Madhu (1994), Dikshit and Sundararajan (1992) and Dixit et al (1995). In the case of lead, copper, magnesium, Al-2024 and Ti-alloy, we used THOR $(1961,1963)$ empirical equation to determine the ballistic performance, which is of the form,

$$
v_{\mathrm{s}}=10^{c}(h)^{\alpha} m_{\mathrm{s}}^{\beta}(\sec \theta)^{\gamma} v_{s}^{\gamma},
$$

where $v_{s}$ is the projectile striking velocity in fps, $h$, the target thickness in inches, $m_{s}$, the weight of the original shot in grains, $\theta$, the angle of obliquity of the impact and $c, \alpha, \beta, \gamma$, $\lambda$ are the material constants for THOR equation.

In all the cases, we have used the results corresponding to a projectile striking velocity of about $800 \mathrm{~m} / \mathrm{sec}$. The material properties and the constants used in THOR equations are presented in tables 1 and 2 respectively. While the calculations of $\alpha_{1}, \alpha_{2}$, $k_{\mathrm{e}}, k_{\mathrm{b}}, k_{\gamma}$, and $k_{\mathrm{j}}$ are simple and straight-forward, it is not the case with $k_{\mathrm{p}}$. Preferably, the speed of the plastic wave should be determined using the slope of the true stressstrain curve, but if such data is not available one can determine using the following equation

$$
\sigma_{\mathrm{u}}^{*}=\sigma_{\mathrm{u}}[1+\% \text { elongation } / 100] \text {, }
$$

or

$$
\sigma_{u}^{*}=\sigma_{u} /(1-\text { reduction in area }) .
$$

The plastic modulus can be determined as,

$$
E_{\mathrm{p}}=\frac{\left(\sigma_{\mathrm{u}}^{*}-\sigma_{\mathrm{y}}\right)}{\% \text { elongation }} \times 100
$$


Table 2. Material constants used for THOR equation.

\begin{tabular}{lccccc}
\hline Material & $\mathrm{C}$ & $\alpha$ & $\beta$ & $\gamma$ & \multicolumn{1}{c}{$\lambda$} \\
\hline Lead & 1.999 & 9.499 & -0.502 & 0.655 & 0.818 \\
Copper & 2.785 & 0.678 & -0.730 & 0.846 & 0.802 \\
Magnesium & 6.904 & 1.092 & -1.170 & 1.050 & -0.087 \\
Al-2024 & 7.047 & 1.029 & -1.072 & 1.251 & -0.139 \\
Ti-alloy & 6.292 & 1.103 & -1.095 & 1.369 & 0.167 \\
\hline
\end{tabular}

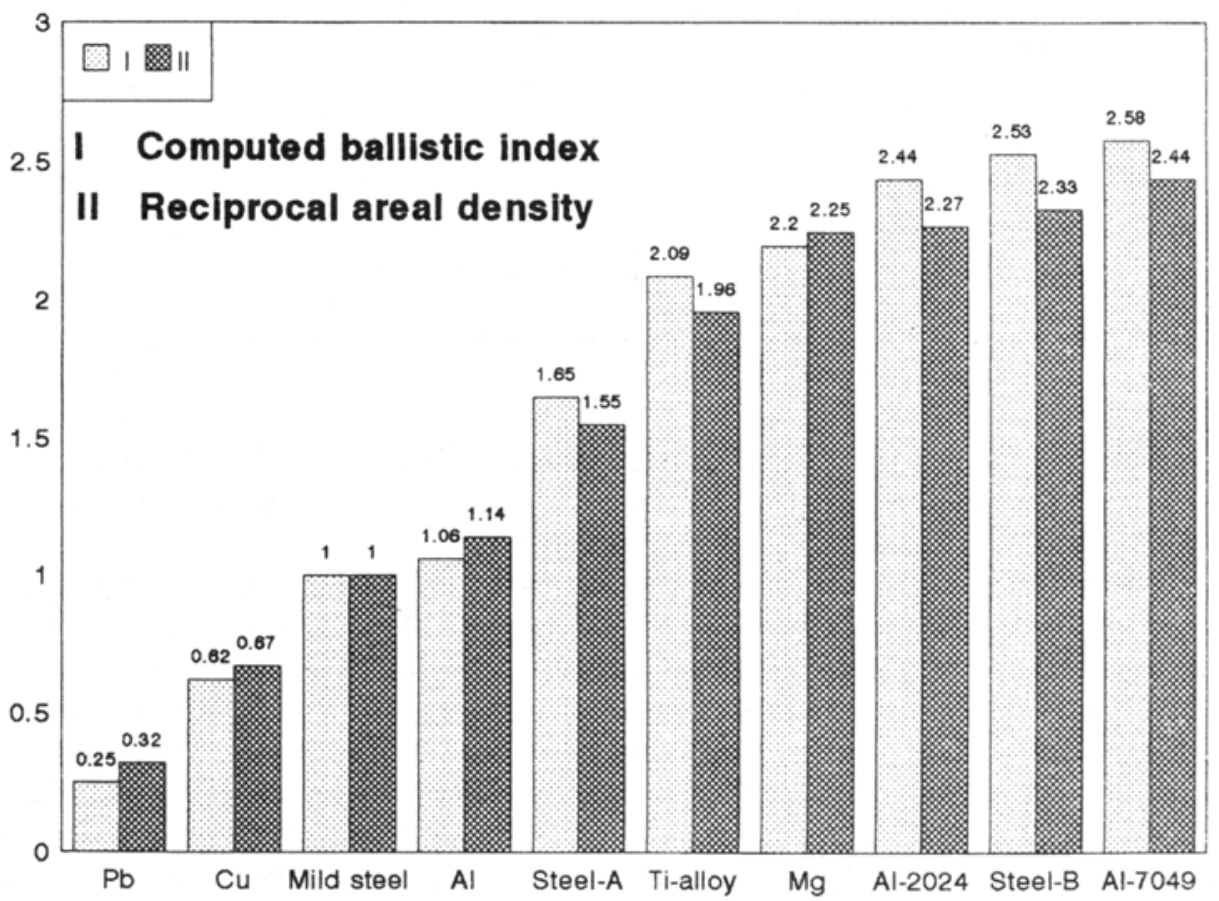

Figure 2. Validation of the ballistic performance index using avallable experimental and empirical results.

For calculating the bulk modulus we have used the standard equation

$$
K=\frac{E}{3(1-2 \gamma)},
$$

where $\gamma$ is the Poisson's ratio.

In figure 2, we have compared the normalized ballistic index with the reciprocal areal density required to provide immunity against a specific threat for a wide range of metallic materials. For lead, the experimental ballistic performance is 0.32 and it is virtually an order of magnitude smaller than that of the aluminum alloy or steel and this variation has been accurately predicted by the index. In addition, even for the alloys of same parent metal, the index can grade the ballistic merit with a reasonable accuracy. 


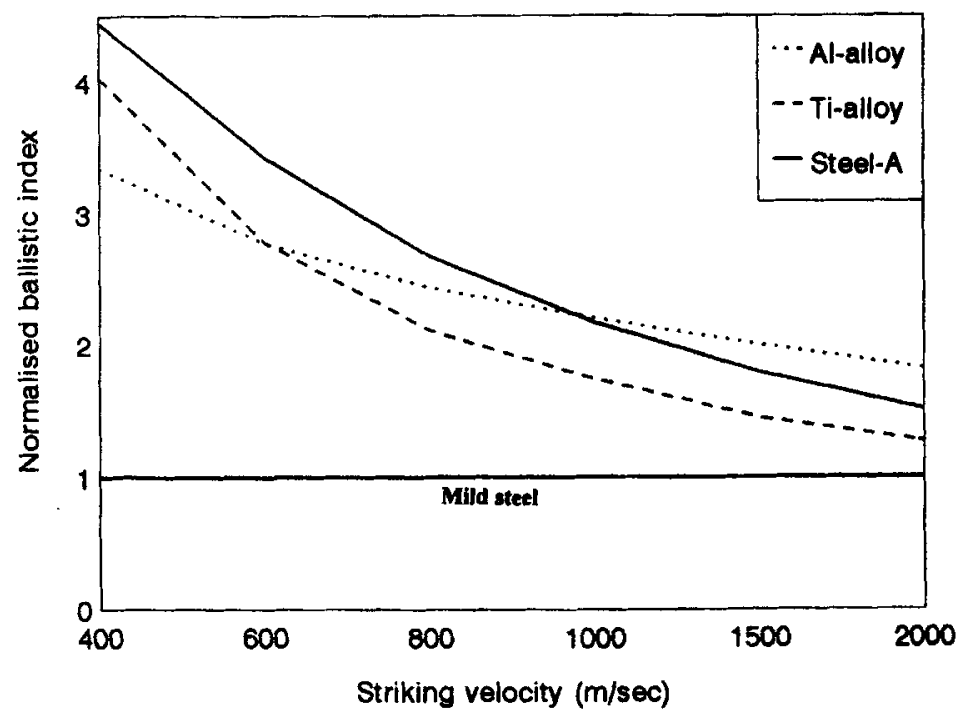

Figure 3. Computed ballistic performance index as a function of striking velocity of the projectile for different materials.

Figure 3 describes the computed index for four different materials shown as a function of the striking velocity. It is interesting to note that the materials such as Al-alloy become superior to steel-B at higher velocities, which is an observed experimental fact (Ramakrishnan 1986). Therefore, the index may find its use in developing even composite metallic armour, where the weight and dimension of the armour can be minimized for a given striking velocity. Another interesting observation is with regard to how the projectile energy is absorbed partly by the deformation mode (elastic and plastic) and partly by the kinetic energy mode. These results are given in figure 4 (a to d) for four different materials. While Ti-alloy and Steel-B show a high cut-off velocity, Al-alloy and mild steel show low cut-off velocities. Using this diagram, it is possible to arrive at a judgement on the efficacy of improving mechanical properties of materials for the purpose of improving the ballistic performance for any specific striking velocity. For instance, figure 4 clearly indicates that on improving the mechanical properties of $\mathrm{Al}$-alloy even by a factor of two would result in an improved performance to a much less extent for a velocity of about $2500 \mathrm{~m} / \mathrm{sec}$. On the other hand, the relevance of the mechanical properties of Ti-alloy is significant even at high velocities.

\section{Summary}

The paper deduces a ballistic performance index for metallic armour materials in terms of the mechanical properties such as strength and modulus. The index is derived using an energy-balance approach where the kinetic energy of the projectile is assumed to be absorbed by the elastic and the plastic deformation involved in the penetration process as well as the kinetic energy imparted to the target material during the deformation. The index is validated using the available experimental and empirical data obtained in the case of a small arm projectile for an impact velocity of about 
$800 \mathrm{~m} / \mathrm{sec}$. The index is useful in estimating the ballistic quality of the metallic material with reasonable accuracy.

The index is given by

$$
\begin{aligned}
\phi= & {\left[\frac{\alpha_{1}}{2\left(1+k_{\mathrm{b}}\right)^{2}}+\alpha_{2} \frac{\left(1+k_{\mathrm{c}}\right)^{2} k_{\gamma}^{2}}{2 k_{\mathrm{j}}^{2}}+\frac{1}{k_{\mathrm{j}}}\left(1+\frac{1}{k_{\mathrm{p}}}\right)+\frac{1}{2 k_{\mathrm{p}}^{2}}\right.} \\
& \left.+\frac{1}{2}\left(1+\frac{1}{k_{\mathrm{p}}}\right)^{2}\right],
\end{aligned}
$$

where $\alpha_{1}$ and $\alpha_{2}$ are the fractional widths of the constrained and the unconstrained zones respectively, these are computed using (3). The non-dimensional parameters $k_{\mathrm{e}}$, $k_{\mathrm{p}}, k_{\mathrm{b}}, k_{\gamma}$, and $k_{\mathrm{j}}$, can be computed as described in (16), (20) and (23). In all the cases $v_{\mathrm{a}}$ is one half of the striking velocity of the projectile.

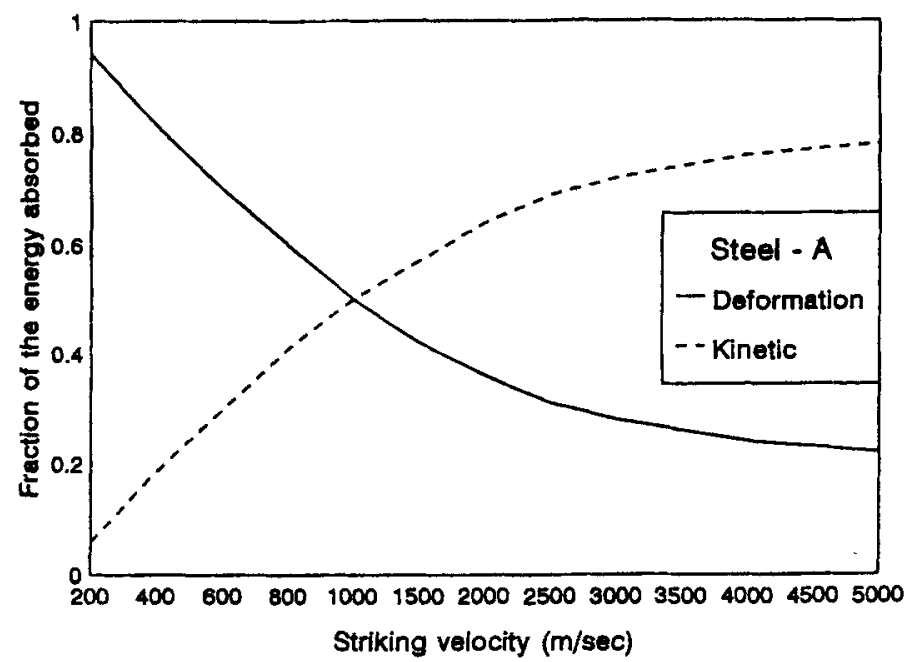

(a)

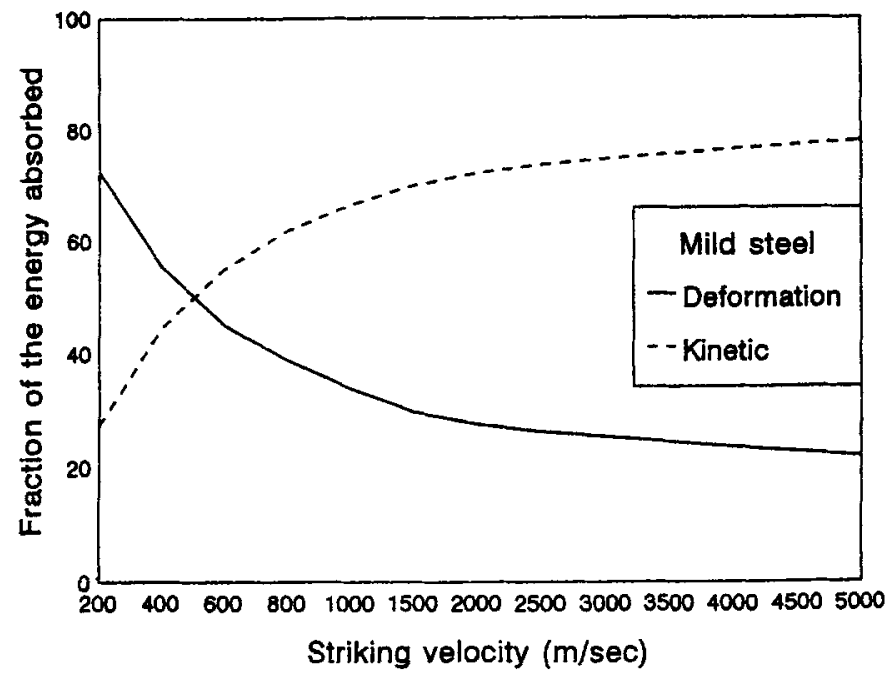

(b)

Figure 4. a-b. 


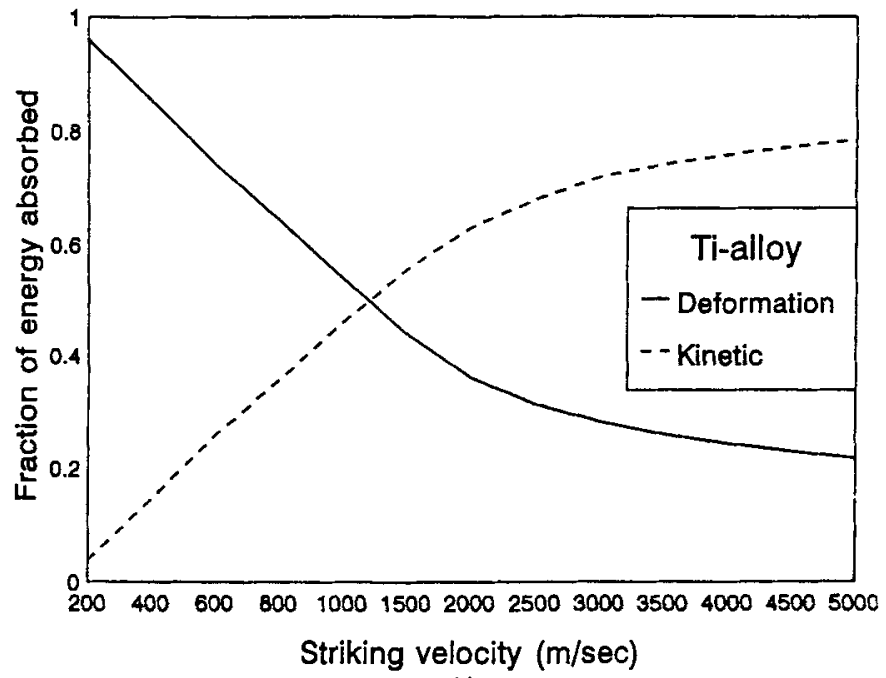

(c)

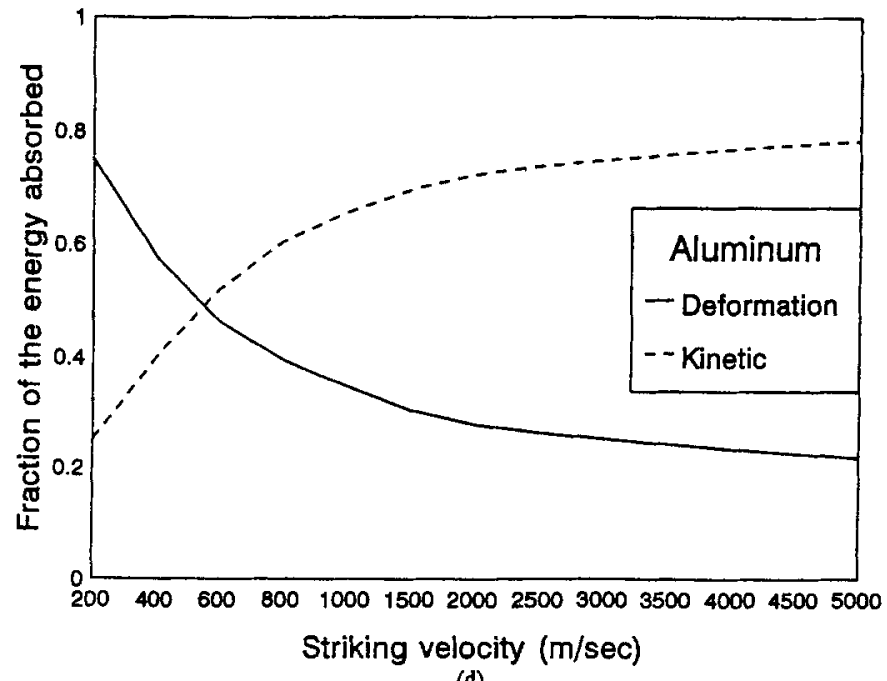

(d)

Figure 4. Fraction of the deformation and kinetic energy components. (a) Steel A, (b) Mild steel, (c) Ti-alloy and (d) aluminium.

\section{Acknowledgement}

The authors thank Col (Dr) S N Dikshit, Dr V Madhu, Dr G Malakondaiah and Dr Trilok Singh for useful discussions.

\section{References}

Abbot K H 1972 J. Mats. 7231

Allen W A and Rogers J W 1961 The Franklin Inst 275

Averbuch J and Bodner S R 1974 Int. J. Solids Struct. 10671 
Backman M E and Goldsmith W 1978 Int. J. Engg. Sci. 161

Bishop R F, Hill R and Molt N F 1945 Proc. Phy. Soc. (London) 53147

Corran R S J, Shadbolt P J and Ruiz C 1983 Int. J. Impact Engg. 13

Dikshit S N and Sundararajan G 1992 Int. J. Impact Engg. 12373

Dikshit S N, Kutumba Rao V V and Sundararajan G 1995 Int. J. Impact Engg. 16293

Eichelberger R J 1956 J. Appl. Phys. 2763

Forrestal M J 1986 Proc. Phys. Soc. 53147

Forrestal M J, Rosenberg Z, Luk V K and Bress S J 1987 Trans. ASME 54230

James D 1987 Int. J. Impact Engg. 5239

Jane G H and Zukas J A 1978 Int. J. Engg. Sci. 5239

Johnson W 1972 Impact strength and materials (London: Edward Arnold Publishers Ltd).

Kinslow R 1970 High-velocity impact phenomena (New York: Academic Press)

Landkof B L and Goldsmith W 1985 Int. J. Solids Struct. 21245

Longcope D B and Forrestal M J 1993 J. Appl. Mech. 50327

Madhu V 1994 Impact of kinetic energy projectiles on single and layered plates, $\mathrm{Ph}$. D. thesis, IIT, Delhi

Metals Handbook ASM 1993 3rd Ed.

Project THOR TR No. 47. 1961 and No. 51. 1963 The resistance of various metallic materials to perforation by steel fragments; empirical relationships for fragment residual velocity and weight. Ballistic Research Laboratory, Aberdeen proving ground, U.S.A

Ramakrishnan N 1986 Ballistic test procedures for armour materials, DMRL Report

Randin J and Goldsmith W 1988 Int. J. Impact Engg. 7229

Ravid M and Bodner S R 1983 Int. J. Engg. Sci. 21577

Recht R F 1964 J. Appl. Mech. 1189

Sinha P, Singh I P and Ma C H 1977 J. Appl, Phys. 48167

Smithells 1983 Metals reference book, 6th Ed.

Tabor D 1948 Proc. R. Soc. A192 247

Tate A 1977 Int. J. Mech. Sci. 19121

Tate A 1980 Int. J. Mech. Sci. 28121

Taylor G I 1948a Q. J. Mech. Appl. Math. 1103

Taylor G I 1948b Proc. R. Soc. London 194289

Thompson M J $1955 \mathrm{~J}$. Appl. Phys. 261

Wingrove A L and Wulf G L 1973 J. Aust. Inst. Metals 18167

Woodward R L 1978 Int. J. Mech. Sci. 20599 